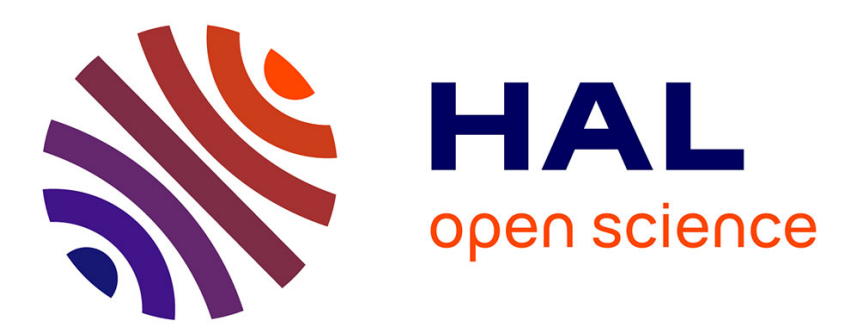

\title{
Physical properties of the complex metallic alloy phases in the Al-Pd-Mn system
}

Janez Dolinsek, Zvonko Jaglicic, Ana Smontara

\section{To cite this version:}

Janez Dolinsek, Zvonko Jaglicic, Ana Smontara. Physical properties of the complex metallic alloy phases in the Al-Pd-Mn system. Philosophical Magazine, 2005, 86 (03-05), pp.671-678. 10.1080/14786430500306519 . hal-00513602

\section{HAL Id: hal-00513602 \\ https://hal.science/hal-00513602}

Submitted on 1 Sep 2010

HAL is a multi-disciplinary open access archive for the deposit and dissemination of scientific research documents, whether they are published or not. The documents may come from teaching and research institutions in France or abroad, or from public or private research centers.
L'archive ouverte pluridisciplinaire HAL, est destinée au dépôt et à la diffusion de documents scientifiques de niveau recherche, publiés ou non, émanant des établissements d'enseignement et de recherche français ou étrangers, des laboratoires publics ou privés. 


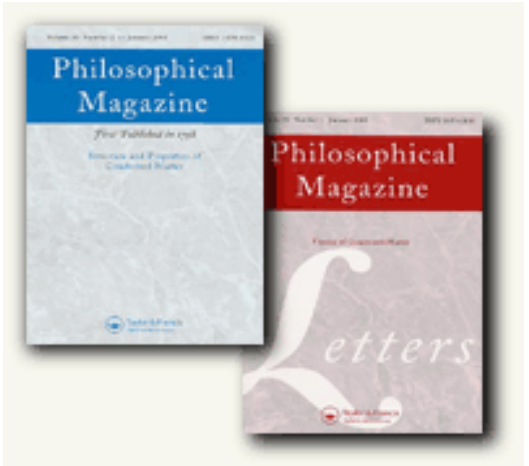

\section{Physical properties of the complex metallic alloy phases in the Al-Pd-Mn system}

\begin{tabular}{|c|c|}
\hline Journal: & Philosophical Magazine \& Philosophical Magazine Letters \\
\hline Manuscript ID: & TPHM-05-Feb-0056.R2 \\
\hline Journal Selection: & Philosophical Magazine \\
\hline $\begin{array}{r}\text { Date Submitted by the } \\
\text { Author: }\end{array}$ & 26-Jul-2005 \\
\hline Complete List of Authors: & $\begin{array}{l}\text { Dolinsek, Janez; J. Stefan Institute, F5 } \\
\text { Jaglicic, Zvonko; Institute of Mathematics, Physics and Mechanics } \\
\text { Smontara, Ana; Institute of Physics }\end{array}$ \\
\hline Keywords: & $\begin{array}{l}\text { transport properties, thermoelectric power, magnetic properties, } \\
\text { aluminium alloys }\end{array}$ \\
\hline Keywords (user supplied): & Complex metallic Alloys, Physical properties, Al-Pd-Mn system \\
\hline
\end{tabular}

\section{今scholarONE" \\ Manuscript Central}




\title{
Physical properties of the complex metallic alloy phases in the Al-Pd-Mn system
}

\author{
J. Dolinšek ${ }^{1}$, Z. Jagličić ${ }^{2}$, A. Smontara ${ }^{3}$ \\ ${ }^{1}$ J. Stefan Institute, University of Ljubljana, Jamova 39, SI-1000 Ljubljana, Slovenia \\ ${ }^{2}$ Institute of Mathematics, Physics and Mechanics, Jadranska 19, SI-1000 Ljubljana, \\ Slovenia \\ ${ }^{3}$ Institute of Physics, Bijenička 46, POB 304, HR-10001 Zagreb, Croatia
}

\begin{abstract}
The Al-Pd-Mn system of intermetallics contains complex metallic alloy (CMA) phases, whose crystal structures are based on giant unit cells comprising up to more than a thousand atoms per cell. We performed investigation of the magnetic, electrical, thermal transport and thermoelectric properties of the $\xi^{\prime}$ phase and the related $\Psi$ phase on singlecrystalline samples.
\end{abstract}

Short title: CMA phases in Al-Pd-Mn system

Keywords: Complex metallic alloys, Physical properties, Al-Pd-Mn system 


\section{INTRODUCTION}

"Complex Metallic Alloys" (CMAs) [1,2] denote intermetallic phases whose giant unit cells with lattice parameters of several nanometers contain from many tens up to more than a thousand atoms. Inside the giant unit cells the atoms are arranged in clusters with polytetrahedral local atomic order of icosahedral or decagonal symmetry as typically found in quasicrystals (QCs). Consequently, in CMAs there exist two substantially different physical length scales - one defined by the cluster substructure and the other by the unit-cell parameters - so that interesting physical properties may appear from the competition between these different length scales.

Among the CMA materials, an interesting family of related structures was revealed in the Al-Pd alloys system $[3,4]$ and its ternaries with transition metals $(\mathrm{Mn}$, Fe, Co, Rh) [5-9]. The simplest regular structure discovered first in the Al-Pd-Mn [5,6] system, designated as $\xi^{\prime}$, contains 320 atoms in its orthorhombic unit cell. Some physical properties of single-crystalline samples of this compound grown by the selfflux technique were already reported [10]. Recently, centimeter-size single crystals of the $\xi^{\prime}$ phase and the related $\Psi$ phase (containing about 1500 atoms in the unit cell) with the composition $\mathrm{Al}_{74} \mathrm{Pd}_{22} \mathrm{Mn}_{4}$ were successfully grown using the Bridgman technique [11]. Here we present a study of their magnetic, electrical, thermal transport and thermoelectric properties.

\section{SAMPLE PREPARATION}

The sample materials were grown by the Bridgman technique, for details see [11]. Three samples were investigated under nominally the same experimental conditions. The samples were selected on the basis of previous TEM examinations [11]. Two 


\section{MAGNETIC MEASUREMENTS}

The magnetization as a function of the magnetic field, $M(H)$, and the temperaturedependent magnetic susceptibility, $\chi(T)$, were measured with a Quantum Design SQUID magnetometer, equipped with a $5 \mathrm{~T}$ magnet. The susceptibility $\chi$ was investigated in the temperature interval between 300 and $2 \mathrm{~K}$ in a magnetic field $H=5$ kOe applied along [010] direction (Fig. 1a). In this field the $M(H)$ dependence is linear (Fig. 1b), so we analyze $\chi=M / H$ in the following. The $\chi(T)$ values of all three samples are negative for the whole of the investigated temperature interval, indicating a dominant diamagnetic contribution. In addition, small paramagnetic Curielike growth of the magnetization upon cooling is observed. The $\chi(T)$ data were analyzed by the sum of a Curie term and a temperature-independent term $\chi_{0}$

$$
\chi=\frac{C}{T-\theta}+\chi_{0}
$$


and the fits are shown as solid lines in Fig. 1a. The values of the parameters $C$ (the Curie constant), $\theta$ (the Curie-Weiss temperature) and $\chi_{0}$ are summarized in Table 1. For an electrically conducting material one generally expects three contributions of comparable size to the temperature-independent term $\chi_{0}$ : the negative Larmor diamagnetic contribution due to closed-shell ion cores, the negative Landau diamagnetic contribution due to orbital motion of conduction electrons in a magnetic field and the positive Pauli spin paramagnetic contribution due to conduction electrons, $\chi_{0}=\chi_{\text {Larmor }}+\chi_{\text {Landau }}+\chi_{\text {Pauli }}$. The core contribution $\chi_{\text {Larmor }}$ can be estimated from Pascal tables [12]. For different choices of the ionization states of the elements (e.g. $\left.\mathrm{Mn}^{2+}, \mathrm{Mn}^{3+}, \mathrm{Mn}^{4+}, \mathrm{Pd}^{3+}, \mathrm{Pd}^{4+}, \ldots\right)$ the calculated $\chi_{\text {Larmor }}$ values are all in the range between $-0.6 \times 10^{-3}$ and $-0.8 \times 10^{-3} \mathrm{emu} / \mathrm{mol}$, whereas the experimental $\chi_{0}$ values are in the interval between $-0.5 \times 10^{-3}$ and $-0.6 \times 10^{-3}$ emu/mol. We, therefore, have $\chi_{0} \approx \chi_{\text {Larmor }}$, so that the diamagnetic contribution of the closed atomic shells dominates the temperature-independent term of the susceptibility. The small, negative values of the Curie-Weiss temperature $\theta$ should be considered as additional fit parameters only, which slightly improve the fits in Fig. 1a. No other experimental results suggest any antiferromagnetic interaction between the magnetic moments. The dominant diamagnetic behaviour is also observed in the $M(H)$ curves (Fig. 1b) obtained at $5 \mathrm{~K}$, which exhibit a typical diamagnetic linear decrease with increasing $H$.

The Curie-type dependence of the paramagnetic part of the magnetization indicates the presence of localized magnetic moments in the structure. In analogy to $i$ Al-Pd-Mn QCs, it is straightforward to attribute this magnetization to the Mn species. 
The small paramagnetic susceptibility, which even at $2 \mathrm{~K}$ is smaller than the diamagnetic core susceptibility, indicates that only a tiny fraction of the Mn atoms are magnetic. The fraction of magnetic moments in the samples can be estimated from the values of the Curie constant $C$, assuming that the magnetic manganese atoms are in the $\mathrm{Mn}^{2+}$ state with the effective Bohr magneton number $p_{\text {eff }}=5.9$. The mean effective Bohr magneton number (the mean $p_{\text {eff }}$ of all the Mn atoms in the sample) is calculated by using the formula [12] $\bar{p}_{\text {eff }}=2.83 \sqrt{C}$, which in turn yields the magnetic fraction as $f=\left(\bar{p}_{\text {eff }} / p_{\text {eff }}\right)^{2}$. The estimated $f$ values of the three investigated samples are given in Table 1. They are very small and of comparable magnitude, all close to $1 \times 10^{-4}$. As the level of extrinsic magnetic impurities in the samples is of the order $10^{-5}$, the measured magnetization contains both the Mn and the impurity moments. Therefore, the $f$ values from Table 1 should be considered as an upper limit for the magnetic Mn fractions in the investigated samples. Similar small magnetic fraction (1 Mn out of every 60000 ) and the conclusion on the significant contribution of impurity moments to the Curie magnetization was obtained also for the $\xi^{\prime}$ flux-grown monocrystals of composition $\mathrm{Al}_{72} \mathrm{Pd}_{25} \mathrm{Mn}_{3}$ studied by Swenson et al. [10]. Comparing magnetic properties of the $\xi^{\prime}$ and $\Psi$ samples to those of the $i$-Al-Pd-Mn QCs, the QCs are generally substantially more magnetic, with the magnetic Mn fractions typically one to two orders of magnitude larger [13], $f \approx 10^{-3}-10^{-2}$.

\section{ELECTRICAL RESISTIVITY}


The electrical resistivity $\rho(T)$ was determined in the temperature interval between 300 and $4 \mathrm{~K}$ using the standard four-terminal technique. The resistivity was measured along $\left[\begin{array}{lll}0 & 1 & 0\end{array}\right]$ direction of the samples and the data are displayed in Fig. 2. The roomtemperature values are $\rho_{300 K}=213 \mu \Omega \mathrm{cm}$ for the $\xi^{\prime}$-AlPdMn-1 sample, $\rho_{300 K}=192 \mu \Omega \mathrm{cm}$ for the $\xi^{\prime}-\mathrm{AlPdMn}-2$ and $\rho_{300 K}=229 \mu \Omega \mathrm{cm}$ for the $\Psi$ AlPdMn. These values are almost the same as those reported previously for the composition $\mathrm{Al}_{72} \mathrm{Pd}_{25} \mathrm{Mn}_{3}$ [10]. The remarkable feature is the very small temperature variation of the resistivity in all three samples: $R=\left(\rho_{300 K}-\rho_{4 K}\right) / \rho_{300 K}=1.4 \%$ for the $\xi^{\prime}-\mathrm{AlPdMn}-1, R=0.5 \%$ for the $\xi^{\prime}-\mathrm{AlPdMn}-2$, and $R=1.7 \%$ for the $\Psi-\mathrm{AlPdMn}$. Such a nearly temperature-compensated resistivity represents an intermediate case between the positive-temperature-coefficient (PTC) resistivity of metals (originating from the scattering of conduction electrons by phonons) and the negative-temperaturecoefficient (NTC) resistivity commonly found in metallic glasses and QCs (due to a gradual electron localization caused by disorder/quasiperiodicity upon cooling). Relating the resistivities of the investigated $\xi^{\prime}$ and $\Psi$ phases to those of the $i$-Al-Pd-Mn QCs, the $\rho$ values of icosahedral samples are one order of magnitude larger (ranging typically from 1000 up to $4000 \mu \Omega c m)$. The $\rho(T)$ of the $i$-Al-Pd-Mn QCs is also drastically different. The resistivities of QCs usually exhibit negative temperature coefficients, but the magnitude of the NTC varies considerably between samples. In addition, the $\rho(T)$ of $i$-Al-Pd-Mn QCs in many cases displays a maximum between room temperature and $4 \mathrm{~K}$ and sometimes also a minimum at still lower temperatures.

\section{THERMAL CONDUCTIVITY}


The thermal conductivities were measured between 8 and $300 \mathrm{~K}$ along [010] direction using an absolute steady-state heat-flow method. The thermal flux through the samples was generated by a $1 \mathrm{k} \Omega \mathrm{RuO}_{2}$ chip-resistor, glued to one end of the sample, while the other end was attached to a copper heat sink. The temperature gradient across the sample was monitored by a chromel-constantan differential thermocouple. The temperature dependence of the thermal conductivity parameter $\kappa(T)$ of all three samples is displayed in Fig. 3. The $\kappa(T)$ values at $280 \mathrm{~K}$ are $8.6 \mathrm{~W} / \mathrm{mK}$ for the $\xi$ AlPdMn-1 sample, $7.3 \mathrm{~W} / \mathrm{mK}$ for the $\xi^{\prime}$-AlPdMn-2 and $5.0 \mathrm{~W} / \mathrm{mK}$ for the $\Psi$-AlPdMn. These low $\kappa(T)$ values are of the same order as those of $i$-Al-Pd-Mn QCs [14], where they are considered to be a consequence of both the low electronic density of states (DOS) at the Fermi energy $E_{F}$ and the nonperiodicity of the lattice, making the electronic and lattice contributions to the heat transport small. The thermal conductivity parameter $\kappa(T)$ is a sum of the electronic and lattice contributions, $\kappa=\kappa_{e l}+\kappa_{l}$. The electronic contribution $\kappa_{e l}$ can be estimated using the Wiedemann-Franz law, $\kappa_{e l}=L_{0} T / \rho$ (where $L_{0}$ is the Lorenz number), and the measured electrical resistivity $\rho(T)$ data from Fig. 2. Due to the very small temperature dependence of $\rho(T)$ that enters the Wiedemann-Franz law, the electronic contribution $\kappa_{e l}(T)$ exhibits practically linear temperature dependence (Fig. 3). Estimating the electronic contribution at $280 \mathrm{~K}$ we find, for the $\xi^{\prime}$-AlPdMn- $1, \kappa_{e l}=3.3 \mathrm{~W} / \mathrm{mK}$, wherefrom we get the lattice contribution $\kappa_{l}=\kappa-\kappa_{e l}=5.3 \mathrm{~W} / \mathrm{mK}$ with the ratio $\kappa_{e l} / \kappa_{l}=0.62$. For the $\xi^{\prime}$-AlPdMn-2 we get $\kappa_{e l}=3.6 \mathrm{~W} / \mathrm{mK}, \quad \kappa_{l}=3.7 \mathrm{~W} / \mathrm{mK} \quad$ and $\kappa_{e l} / \kappa_{l}=0.97$, whereas for the $\Psi$-AlPdMn we get $\kappa_{e l}=3.0 \mathrm{~W} / \mathrm{mK}$, 
$\kappa_{l}=2.0 \mathrm{~W} / \mathrm{mK}$ and the ratio $\kappa_{e l} / \kappa_{l}=1.5$. For all three samples, $\kappa_{e l}$ and $\kappa_{l}$ are thus of comparable size at room temperature. This is different from both simple metals, where the electronic contribution is usually 1-2 orders of magnitude larger than the lattice contribution, and Al-based QCs, where electrons carry less than $1 \%$ of the heat.

\section{THERMOELECTRIC POWER}

The thermopower measurements were performed at temperatures between 300 and $4 \mathrm{~K}$ by applying a differential method with two identical thermocouples (chromel-gold with $0.07 \%$ iron), attached to the sample with silver paint. The thermoelectric power data (the Seebeck coefficient $S(T)$ ) are shown in Fig. 4. The thermopowers of all three investigated samples are negative across the whole investigated temperature interval, indicating that electrons are the dominant charge carriers. The room-temperature $S$ values are rather small, ranging between -4.7 and $-8 \mu \mathrm{V} / K$. While the $\xi$ '-AlPdMn-1 and $\xi^{\prime}$-AlPdMn-2 samples exhibit a relatively smooth temperature variation of $S(T)$, the thermopower of the $\Psi$-AlPdMn sample shows a strong (absolute) increase between $4 \mathrm{~K}$ and $8 \mathrm{~K}$, followed by a weaker increase at higher temperatures. For all samples, $S(T)$ shows several changes of slope within the investigated temperature range.

\section{CONCLUSIONS}

We investigated magnetic, electrical, thermal transport and thermoelectric properties of the giant-unit-cell $\xi^{\prime}$ and $\Psi$ CMA phases in the Al-Pd-Mn system. Magnetic susceptibility measurements have shown that the samples are diamagnets with a tiny paramagnetic Curie-like magnetization. The estimated fraction of magnetic Mn atoms 
(assumed to be in the $\mathrm{Mn}^{2+}$ state) in all samples is about $1 \times 10^{-4}$. The electrical resistivity amounts about $200 \mu \Omega \mathrm{cm}$ and is temperature-independent to within less than $2 \%$ in the investigated interval between 300 and $4 \mathrm{~K}$. The thermal conductivity of the $\xi$ ' and $\Psi$ samples is small, comparable in magnitude to that of $i$-Al-Pd-Mn QCs. This suggests that a low electronic DOS at the $E_{F}$ and a low phonon density are at the origin of the weak heat transport in the $\xi^{\prime}$-Al-Pd-Mn CMAs. The thermoelectric power of about $-6 \mu \mathrm{V} / \mathrm{K}$ at room temperature is small, compatible with a low concentration of charge carriers, and negative, indicating that electrons are the majority carriers.

Acknowledgement: We thank M. Feuerbacher for provision of the samples. 
Table 1: Parameters $C, \theta$ and $\chi_{0}$ obtained from the fits of the temperature-dependent magnetic susceptibility. The magnetic fraction $f$ of $\mathrm{Mn}$ atoms was calculated from the Curie constants $C$, assuming the atoms are in the $\mathrm{Mn}^{2+}$ state $\left(p_{\text {eff }}=5.9\right)$.

\begin{tabular}{|c|c|c|c|c|}
\hline sample & $C$ & $\theta$ & $\chi_{0}$ & $f$ \\
& $(\mathrm{emu} \mathrm{K}) / \mathrm{mol} \mathrm{Mn}$ & $(\mathrm{K})$ & $\left(\times 10^{-3} \mathrm{emu} / \mathrm{mol}\right.$ sample $)$ & \\
\hline$\xi^{\prime}$-AlPdMn-1 & $3.0 \times 10^{-3}$ & -3.0 & -0.59 & $0.7 \times 10^{-4}$ \\
\hline$\xi^{\prime}$-AlPdMn-2 & $4.7 \times 10^{-3}$ & -3.2 & -0.56 & $1.0 \times 10^{-4}$ \\
\hline$\Psi-$ AlPdMn & $6.9 \times 10^{-3}$ & -5.4 & -0.52 & $1.6 \times 10^{-4}$ \\
\hline
\end{tabular}




\section{References:}

[1] The term "Structurally Complex Alloy Phases" or SCAPs is also sometimes used in literature.

[2] See, for a recent review, K. Urban and M. Feuerbacher, J. Non-Cryst. Solids 334\&335, 143 (2004).

[3] Y. Matsuo, K. Hiraga, Phil. Mag. Lett. 70, 155 (1994).

[4] M. Yurechko, A. Fattah, T. Velikanova, B. Grushko. J. Alloys Comp. 329, 173 (2001).

[5] M. Boudard, H. Klein, M. de Boissieu, M. Audier, H. Vincent, Philos. Mag. A 74, 939 (1996).

[6] H. Klein, M. Audier, M. Boudard, M. de Boissieu, L. Behara, M. Duneau, Philos. Mag. A 73, 309 (1996).

[7] S. Balanetskyy, B. Grushko, T. Ya. Velikanova, K. Urban, J. Alloys Comp. 376, 158 (2004).

[8] M. Yurechko, B. Grushko, T. Velikanova, K. Urban, J. Alloys Comp. 337, 172 (2002).

[9] S. Balanetskyy, B. Grushko, T. Ya. Velikanova, Z. Kristall. 219, 548 (2004).

[10] C. A. Swenson, I. R. Fisher, N. E. Anderson, Jr., P. C. Canfield, A. Migliori, Phys. Rev. B 65, 184206 (2002).

[11] M. Feuerbacher, C. Thomas, K. Urban, in Quasicrystals, Structure and Physical Properties, Ed. H.-R. Trebin (Wiley-VCH, Weinheim, 2003), p. 2.

[12] F. E. Mabbs, D. J. Machin, Magnetism and Transition Metal Complexes (Chapman and Hall, London, 1973), p. 7. 
[13] F. Hippert, M. Audier, J. J. Préjean, A. Sulpice, E. Lhotel, V. Simonet, Y. Calvayrac, Phys. Rev. B 68, 134402 (2003).

[14] A. Bilušić, Ž. Budrović, A. Smontara, J. Dolinšek, P. C. Canfield, I. R. Fisher, J. Alloys. Compd. 342, 413 (2002). 


\section{Figure captions:}

Fig. 1: (a) Temperature-dependent magnetic susceptibility $\chi$ in a field $H=5 \mathrm{kOe}$ (solid lines are fits described in the text) and (b) magnetization $M$ as a function of the magnetic field $H$ at $\mathrm{T}=5 \mathrm{~K}$ (the slight nonlinearity in the vicinity of $H=0$ is due to a thin ferromagnetic surface oxide layer). The dashed lines serve as a guide for the eye and "f. u." denotes "formula unit" (e.g. one $\mathrm{Al}_{73} \mathrm{Pd}_{22.9} \mathrm{Mn}_{4.1}$ unit in the case of $\xi^{\prime}-$ AlPdMn-1 sample).

Fig. 2: Temperature-dependent electrical resistivities.

Fig. 3: Temperature-dependent thermal conductivities $\kappa(T)$ between 8 and $300 \mathrm{~K}$. Squares, circles and triangles represent the total conductivities, whereas the dotted, dashed and dash-dot lines represent the electronic contribution $\kappa_{e l}$, estimated from the Wiedemann-Franz law.

Fig. 4: Thermoelectric power $S(T)$ between 4 and $300 \mathrm{~K}$. 

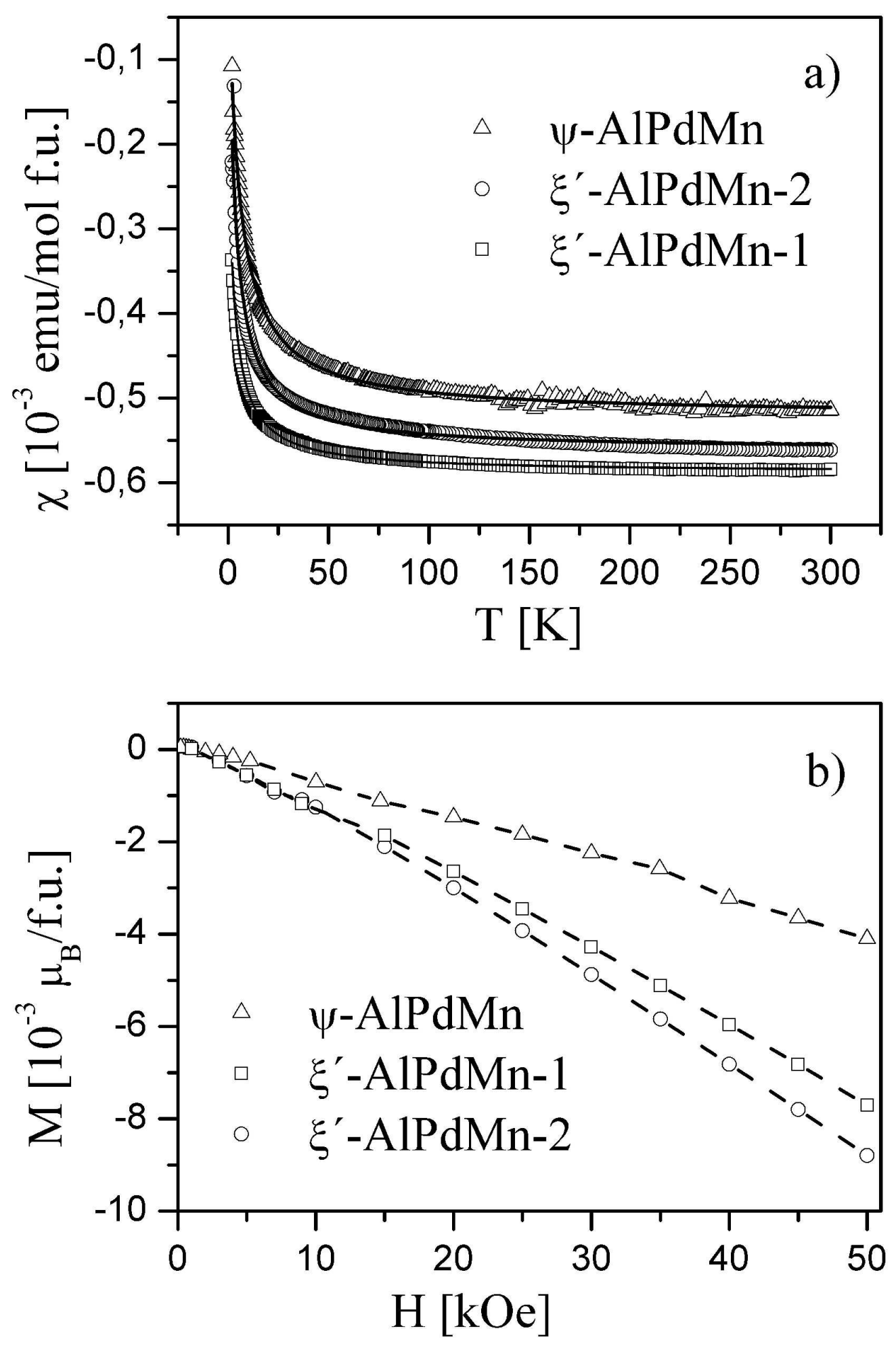

Fig. 1 


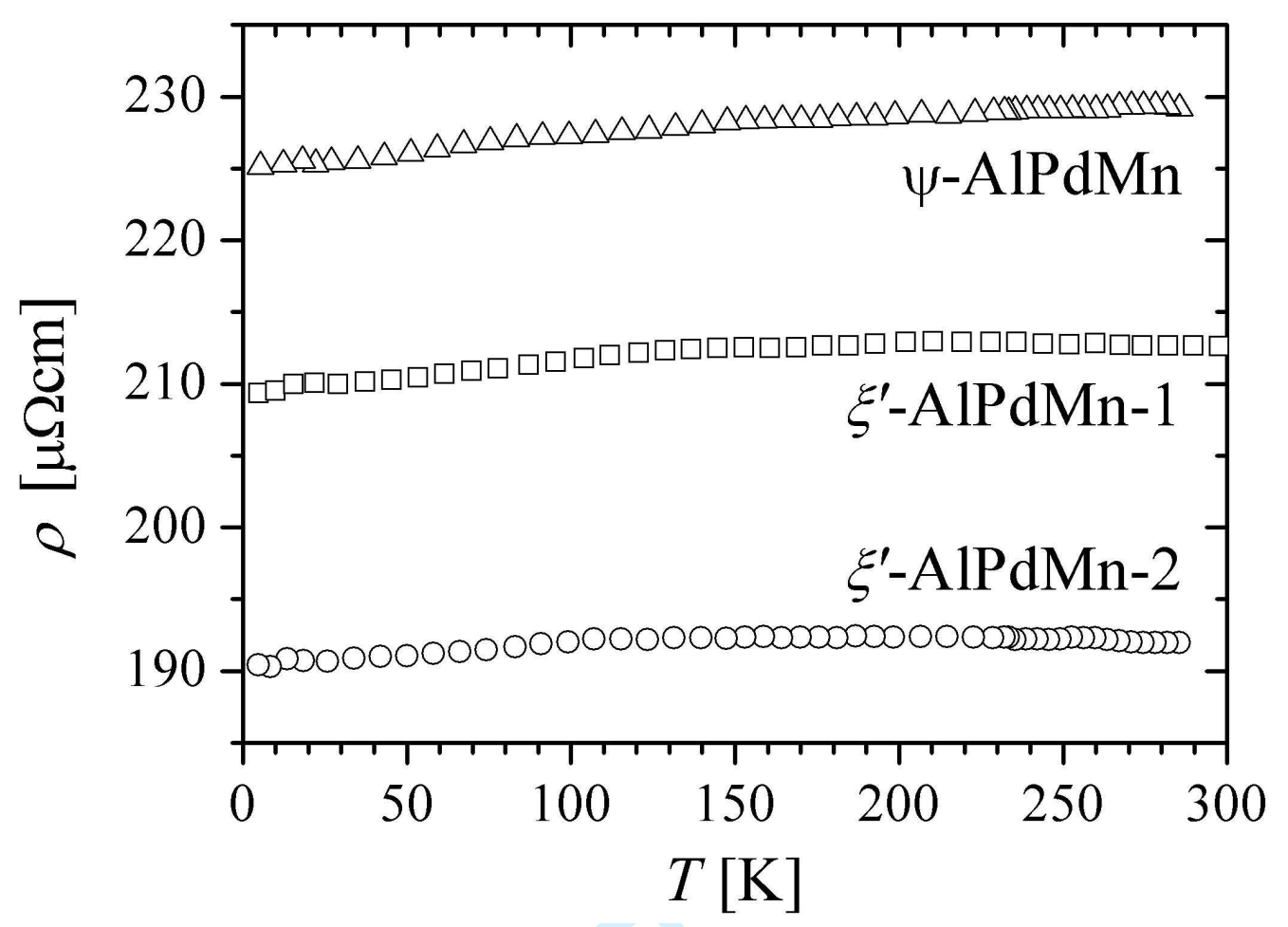

Fig. 2 


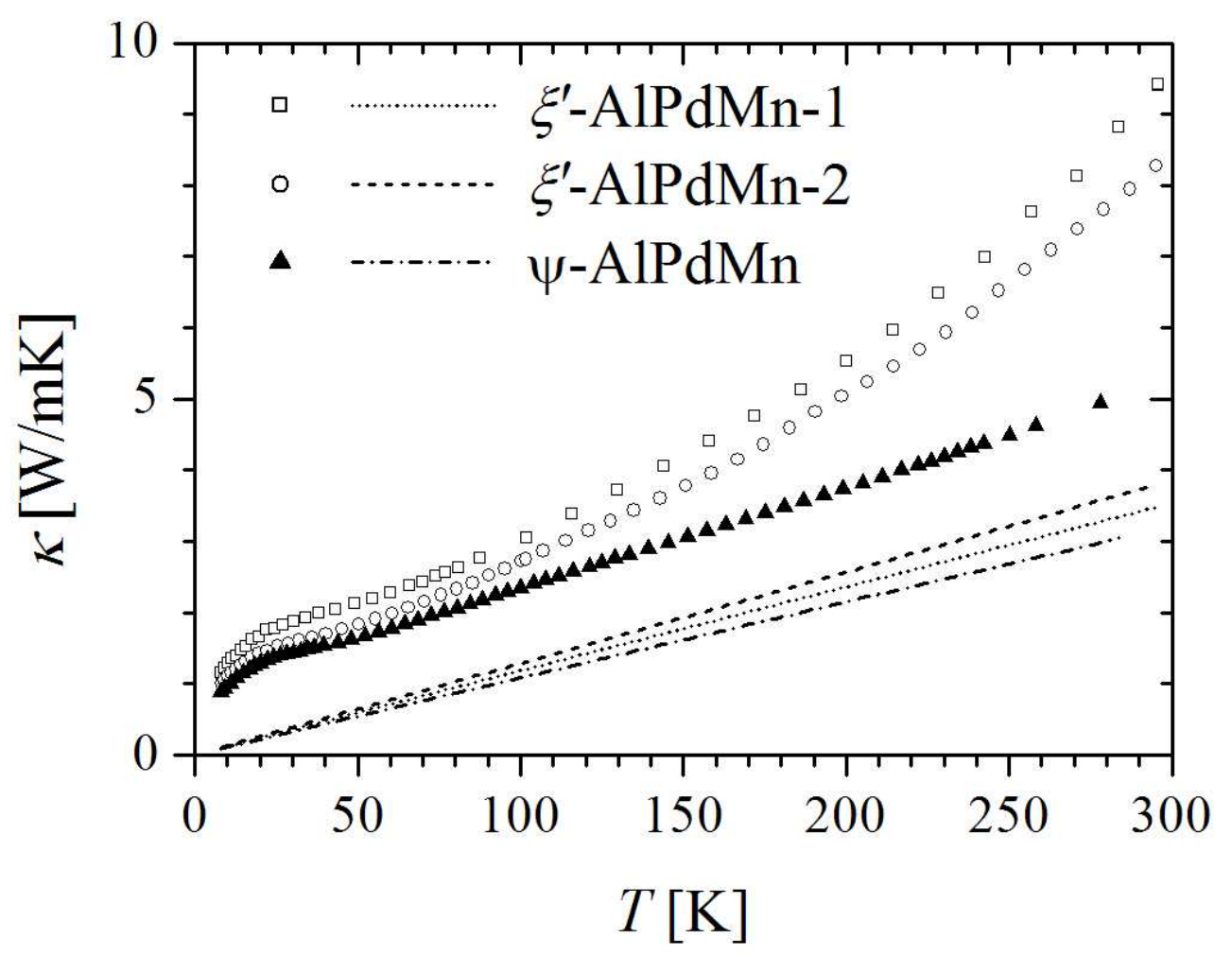

Fig. 3 


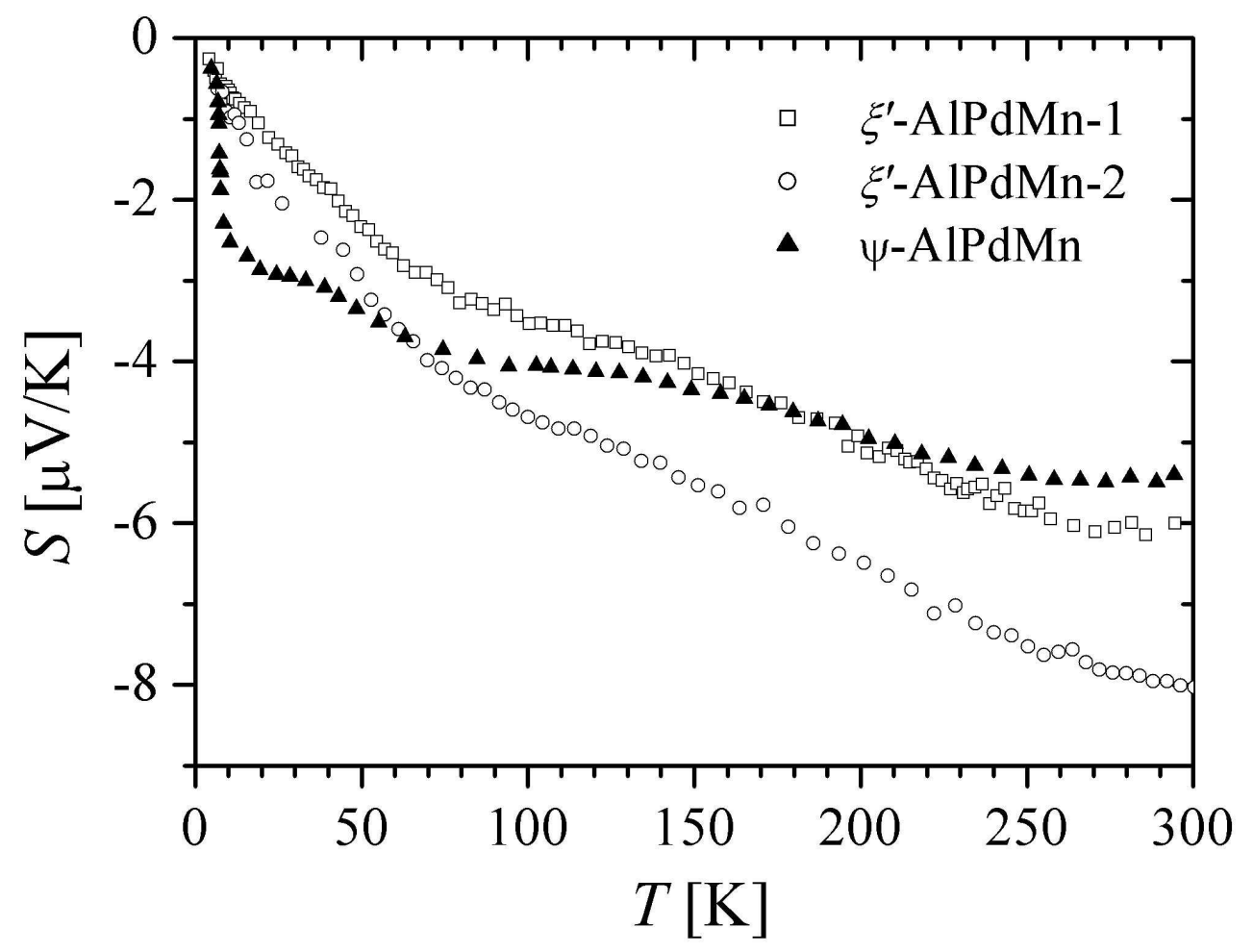

Fig. 4 